\title{
El registro más antiguo de Panochthus (Xenarthra, Glyptodontidae)
}

\section{The oldest record of Panochthus (Xenarthra, Glyptodontidae)}

\author{
M. Zamorano ${ }^{1}$, M. Taglioretti' ${ }^{2}$ A.E. Zurita ${ }^{3}$, G.J. Scillato-Yané ${ }^{1}$, Y.F. Scaglia ${ }^{4}$ \\ ${ }^{1}$ División Paleontología de Vertebrados, Museo de La Plata, Facultad de Ciencias Naturales y Museo, Universidad Nacional de \\ La Plata. Paseo del Bosque s/n, 1900 La Plata. Argentina. CONICET. Email: marzamorano@fcnym.unlp.edu.ar \\ Instituto de Geología de Costas y del Cuaternario. Facultad de Ciencias Exactas y Naturales, Universidad Nacional de Mar \\ del Plata, Buenos Aires, Argentina \\ ${ }^{3}$ Centro de Ecología Aplicada del Litoral (CECOAL-CONICET). Ruta 5, km 2.5, 3400 (CC 128) Corrientes, Argentina \\ ${ }^{4}$ Museo Municipal de Ciencias Naturales de Mar del Plata "Lorenzo Scaglia". Mar del Plata, Buenos Aires
}

\section{RESUMEN}

Panochthus es uno de los géneros de gliptodóntidos de mayores dimensiones y más ampliamente registrado en el Pleistoceno del sur de América del Sur. Una reciente revisión demostró que alberga seis especies distribuidas cronológicamente de la siguiente manera: $P$. intermedius y $P$. subintermedius (Pleistoceno tempranoPleistoceno medio, Ensenadense); $P$. tuberculatus y $P$. frenzelianus tienen sus biocrones limitados al Pleistoceno medio-Pleistoceno tardío (Bonaerense-Lujanense), mientras que $P$. greslebini y $P$. jaguaribensis solo pueden referirse al Pleistoceno sensu lato. En la presente contribución se da a conocer el registro más antiguo de Panochthus, representado por el material MMP 5171, procedente del Plioceno tardío (Aloformación Playa Los Lobos, Formación Chapadmalal; Chapadmalalense; Biozona de Paraglyptodon chapalmalensis) de la localidad de Mar del Plata, Buenos Aires, Argentina. El material consiste en un gran fragmento de coraza dorsal y en varios trozos de osteodermos asociados, asignables a una especie indeterminada de Panochthus. Uno de los caracteres más significativos es la presencia, a nivel de la superficie expuesta de los osteodermos, de figuritas que siguen un evidente patrón reticular, solo observable en Panochthus. Desde el punto de vista bioestratigráfico, es llamativa la ausencia de registros de Panochthus en la Edad Marplatense (Plioceno tardío-Pleistoceno temprano).

Palabras clave: Panochthus; Chapadmalalense; Plioceno; Pleistoceno; Argentina

\begin{abstract}
Panochthus is one of the largest sized and most frequently recorded genera of Pleistocene South American Glyptodontidae. A recent taxonomic revision shows that the genus includes six species: $P$. intermedius and $P$. subintermedius (early Pleistocene-middle Pleistocene), $P$. tuberculatus and $P$. frenzelianus (middle Pleistocene-late Pleistocene), whereas $P$. greslebini and $P$. jaguaribensis only can be referred to the Pleistocene sensu lato. In this contribution the oldest record of the genus Panochthus (MMP 5171) is presented and described. The remains came from the late Pliocene (Playa Los lobos Aloformation, Chapadmalal Formation; Chapadmalalan; Paraglyptodon chapalmalensis Biozone) of the Mar del Plata, Buenos Aires province, Argentina. The material is represented by a considerable fragment of dorsal carapace and some associated osteoderms, which are assignable to an indeterminate species of Panochthus. One of the most significant characters in the exposed surface of the osteoderms is the presence of a clear "reticular" pattern, a character only observed in Panochthus. From a biostratigraphic viewpoint, it is remarkable the absence of records of Panochthus in the Marplatan Age/Stage (late Pliocene-early Pleistocene).
\end{abstract}

Keywords: Panochthus; Chapadmalalan; Pliocene; Pleistocene; Argentina 


\section{Introducción}

Panochthus esungénerode gliptodonte(Xenarthra, Glyptodontidae) reconocido por Burmeister (1866), el cual fue originalmente descripto como un subgénero de Glyptodon Owen 1839 (Burmeister, 1866: 190-191). Constituye asimismo uno de los gliptodontes pleistocenos de tamaño gigantesco (ScillatoYané \& Carlini, 1998; Fariña, 2000; Soibelzon et al., 2012), y uno de los que poseen mayor abundancia de registros en la región Pampeana (Scillato-Yané et al., 1995; Cione et al., 1999; Zamorano, 2012a). Junto con Glyptodon Owen son los gliptodontes de mayor distribución latitudinal en América del Sur (Zurita et al., 2009; Zamorano, 2012a). Previamente a esta contribución se registraba desde el Ensenadense (Pleistoceno temprano) hasta el Lujanense (hasta ahora solo en el lapso Pleistoceno tardío de esta Edad) en Argentina, Uruguay, Brasil y Bolivia (Zamorano, 2012a, 2012b).

En una reciente revisión Zamorano (2012a, 2012b) demostró que el género alberga seis especies distribuidas cronológicamente de la siguiente manera: $P$. intermedius y $P$. subintermedius se registran en el lapso Pleistoceno temprano-Pleistoceno medio (Ensenadense), en tanto que $P$. tuberculatus y $P$. frenzelianus tienen sus biocrones limitados al Pleistoceno medioPleistoceno tardío (Bonaerense-Lujanense). Las restantes especies de Panochthus (P. greslebini y $P$. jaguaribensis) pueden referirse al Pleistoceno sensu lato (ver Ameghino, 1889; Lydekker, 1895; Castellanos, 1937, 1942; Moreira, 1965, 1971; Bergqvist, 1993). Porpino \& Bergqvist (2002) registran materiales de estas dos especies para el Pleistoceno Tardío.

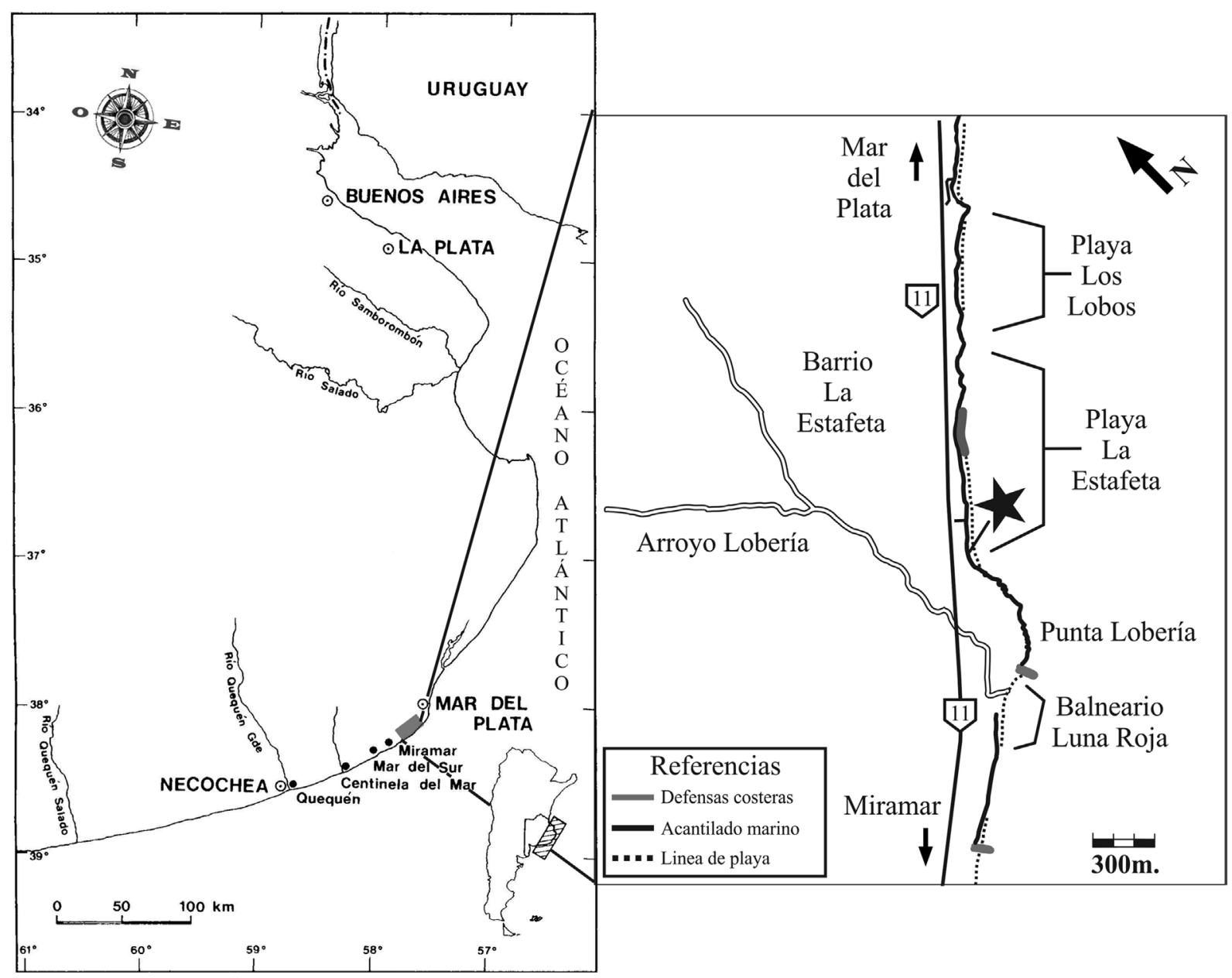

Fig 1.-Mapa que muestra la localización geográfica de Panochthus (MMP 5171). 


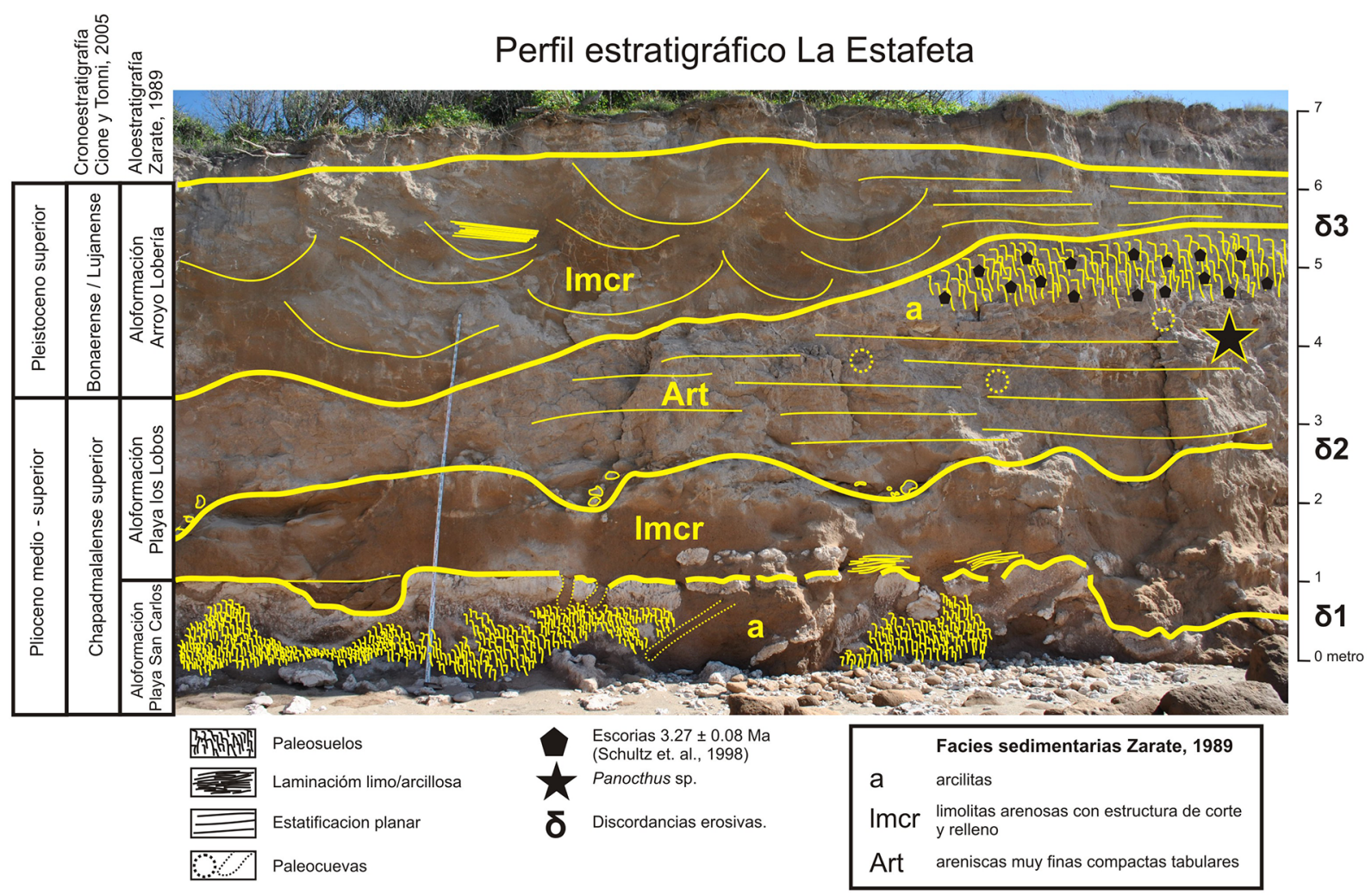

Fig 2.-Perfil estratigráfico que muestra la procedencia del material MMP 5171.

En la presente contribución se da a conocer el registro más antiguo de restos de una especie indeterminada de Panochthus. El material (MMP 5171) está representado por un fragmento considerable de la región póstero-dorsal de la coraza dorsal y varios fragmentos constituidos por osteodermos asociados. Procede del Plioceno tardío (Aloformación Playa Los Lobos, Formación Chapadmalal; Chapadmalalense; Biozona de Paraglyptodon chapalmalensis) de Playa La Estafeta, Mar del Plata (38 $\left.10^{\prime} 3.08^{\prime \prime} \mathrm{S} / 5^{\circ} 38^{\prime} 4.69^{\prime \prime} \mathrm{O}\right)$ (Fig. 1; Fig. 2).

\section{Descripción y comparaciones}

El material consiste en un fragmento considerable de coraza dorsal de $390 \mathrm{~mm}$ por $410 \mathrm{~mm}$, correspondiente a la región póstero-dorsal de la coraza dorsal, separado en dos porciones más grandes, y en varios trozos de osteodermos asociados de la misma estructura (Fig. 3A). En vista interna, se observan restos de la cintura pélvica. Ciertos sectores de la coraza se encuentran deteriorados, a pesar de lo cual puede observarse claramente el patrón de ornamentación.
Los osteodermos son poligonales, en su mayoría hexagonales. La porción de coraza más grande presenta osteodermos de $54,5 \mathrm{~mm}$ de diámetro anteroposterior por $44 \mathrm{~mm}$ de diámetro transverso; el espesor de esta porción es de 18 a $20 \mathrm{~mm}$. En la porción de coraza menor, los osteodermos miden $62 \mathrm{~mm}$ de diámetro antero-posterior por $46 \mathrm{~mm}$ de diámetro transverso; en esta porción el espesor es mayor, alcanzado los $24 \mathrm{~mm}$.

La ornamentación de la superficie expuesta consiste en figuritas poligonales pequeñas, de superficie plana y aspecto rugoso, con tamaños de entre 5 a $10 \mathrm{~mm}$, distribuidas por toda la superficie; en al menos dos osteodermos es posible observar una figurita subcentral, subcircular, ligeramente mayor a las periféricas $(\sim 12 \mathrm{~mm})$, cuya superficie se encuentra deprimida con respecto a la de las figuritas que la rodean. En estos casos se pueden contar de 3 a 4 hileras de figuritas periféricas, pero estas hileras solo pueden apreciarse en un sector del osteodermo debido a la posición subcentral de la figura central. En los surcos que separan las figuritas poligonales es posible observar varias perforaciones e incluso 
también sobre estos. Por otra parte existen figuritas compartidas entre osteodermos contiguos. En $P$. tuberculatus, $P$. subintermedius y $P$. frenzelianus se observa este mismo patrón reticular (Fig. 3C, 3E, 3F). En el único fragmento de coraza preservado (región dorsal según Moreira (1971) y Porpino \& Bergqvist (2002)) de P. jaguaribensis, y en las regiones antero-dorsal y póstero-dorsal de $P$. intermedius, los osteodermos presentan una figura central distinguible, rodeada de varias hileras de figuritas periféricas; en el resto de la coraza de $P$. intermedius se observan figuritas distribuidas formando un patrón reticular (Fig. 3D).

Cabe señalar que las figuritas de los osteodermos de $P$. tuberculatus y $P$. frenzelianus (ambas del Bonaerense y Lujanense) son más pequeñas, prácticamente en cualquier región de la coraza, que aquellas de $P$. intermedius y $P$. subintermedius (ambas del Ensenadense). El ejemplar del Chapadmalalense posee figuritas relativamente grandes, comparables a aquellas de las dos últimas especies citadas.
Comparado con los restos conocidos de Propanochthus bullifer (Burmeister) ("Brocherense", Mioceno tardío-Plioceno?) las diferencias son evidentes, ya que en la región póstero-dorsal de la coraza de esta especie existe, en cada osteodermo, una gran figura central rodeada de entre tres y siete hileras de figuritas periféricas. Por su parte, en Nopachtus Ameghino (N. cabrerai Zamorano, Scillato-Yané, González Ruiz \& Zurita y N. coagmentatus Ameghino; Mioceno tardío-Plioceno?) es posible observar la presencia de una figura central rodeada de una o dos hileras de figuritas periféricas angulosas, cuyo número varía de acuerdo a las diferentes áreas de la coraza (ver Zamorano et al., 2011).

A su vez, Plohophorus figuratus, Propanochthus y Nopachtus presentan en cada osteodermo, una figura central subcircular rodeada de varias hileras de figuritas periféricas subcirculares.

Neuryurus trabeculatus (Lujanense de Entre Ríos, Argentina) presenta, en los osteodermos de mayor espesor de la región dorsal, pequeños tubérculos
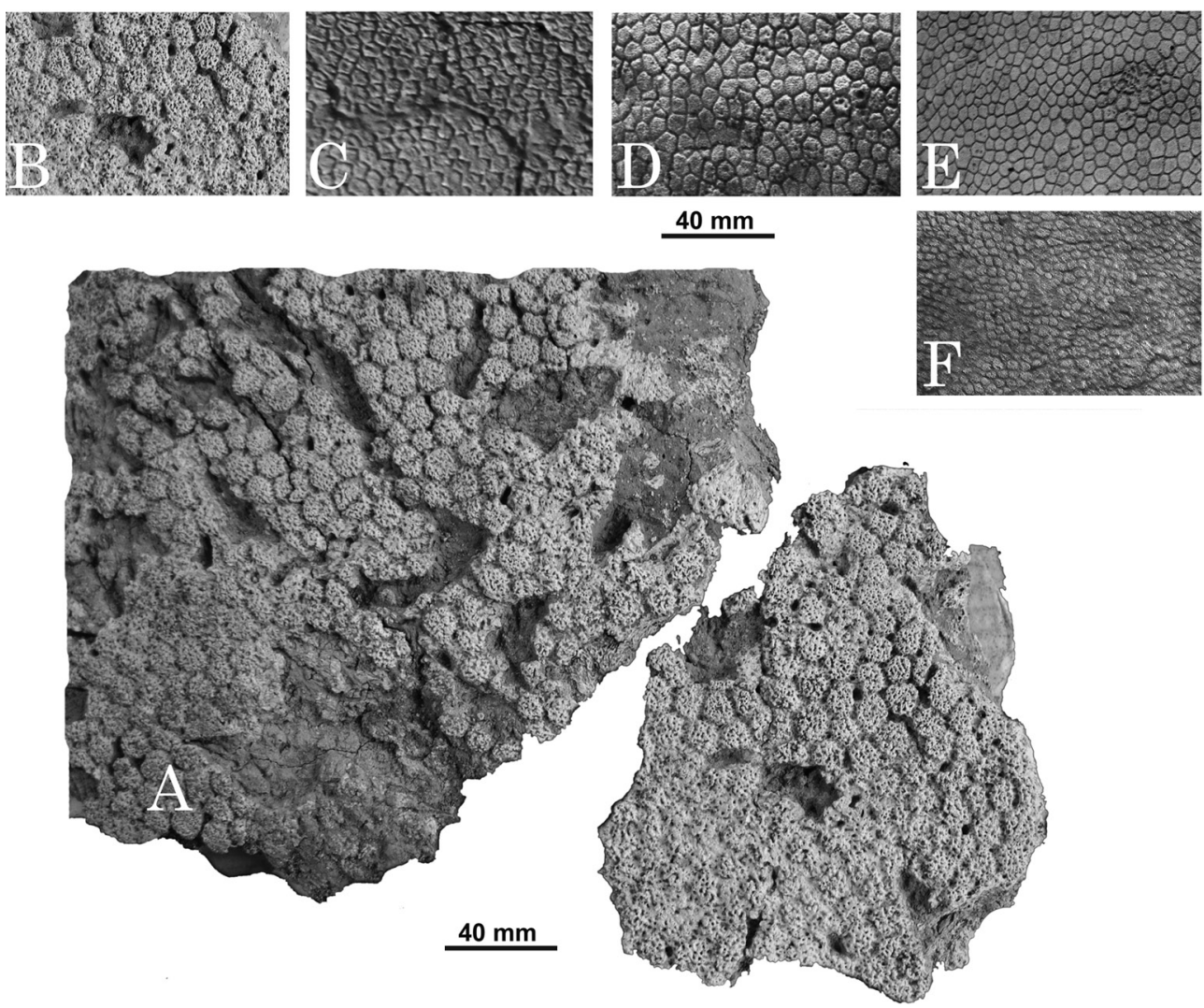

Fig. 3-A-B, fragmentos de la región póstero-dorsal de la coraza dorsal de Panochthus sp. (MMP 5171). C, P. tuberculatus (MLP 16-29). D, P. intermedius (MLP 16-36). E, P. subintermedius (MACN-Pv 5130). F. P. frenzelianus (AMNH 11243). 
distribuidos uniformemente en toda la superficie, entre los cuales existen forámenes (Zurita \& Ferrero, 2009). En Panochthus, los tubérculos presentan cierta semejanza con los de la especie anterior, pero están mejor delimitados por surcos y no siempre presentan forámenes, y si hay no son tan numerosos.

Dentro de los Glyptodontinae, Glyptodon reticulatus también presenta una figura central en todos los osteodermos de la coraza dorsal, pero con la particularidad de que en la región dorsal se observa, en algunos ejemplares, un patrón reticular, de tal manera que, en una primera instancia, resulta difícil identificar los límites de los osteodermos. Sin embargo, en esta especie, las figuritas son siempre notablemente mayores que las del ejemplar MMP 5171, así como de las de todas las especies de Panochthus.

\section{Conclusiones}

En todas las especies de Panochthus, excepto en $P$. jaguaribensis, y en la regiones anteriormente mencionadas de $P$. intermedius (Lydekker, 1895; Castellanos, 1942; Zurita et al., 2011a; Zamorano, 2012a), los osteodermos de las regiones dorsal y póstero-dorsal de la coraza dorsal muestran las figuritas, bien delimitadas por surcos, dispuestas en un claro patrón reticular, un rasgo característico, aunque no exclusivo, de Panochthus (ver Fig. 3) (Moreira, 1971; Bergqvist, 1993; Porpino \& Bergqvist, 2002; Zamorano, 2012a). En este contexto, sería imprudente atribuir el material pertinente a una especie determinada de Panochthus.

Desde el punto de vista crono-estratigráfico, el registro más antiguo del género Panochthus estaba limitado al Piso/Edad Ensenadense (Pleistoceno temprano-Pleistoceno medio) (P. intermedius y P. subintermedius); sin embargo, el nuevo registro amplía la distribución estratigráfica para el género, ya que el hallazgo proviene del Piso/Edad Chapadmalalense (Plioceno tardío). En este sentido, resulta llamativa la ausencia de registros de Panochthus en la Edad Marplatense (Plioceno tardío-Pleistoceno temprano), ya que resulta notable el hiato de circa 1,5 Ma. existente en el registro de Panochthus (Scillato-Yané et al., 1995 y Cione \& Tonni, 2005).

Los Glyptodontidae del Marplatense constituyen un grupo escasamente conocido, en tanto la mayoría de los registros están limitados a restos aislados de la coraza dorsal y/o caudal (Cione et al., 1999; Zurita et al., 2011b), correspondientes a los géneros Hoplophractus Cabrera, Trachycalyptus Ameghino, Urotherium Castellanos, Plohophorus Ameghino y Neosclerocalyptus Paula Couto (Scillato-Yané, et al., 1995).

Scillato-Yané, et al. (1995) sugieren, por la asociación de los registros de xenartros, que las condiciones climáticas de la Edad Chapadmalalense han sido áridas o semiáridas; a su vez, la presencia de Nothotheriinae, parcialmente folívoros, indica la existencia de formaciones arbóreas (seguramente poco extendidas).

\section{AGRADECIMIENTOS}

Los autores desean expresar su agradecimiento al personal del Museo de Ciencias Naturales de Mar del Plata "Lorenzo Scaglia" por permitirnos el acceso al estudio del ejemplar aquí presentado. A D. Perea y E.V: Oliveira, por sus valiosos aportes que permitieron mejorar este trabajo. Trabajo parcialmente financiado a través los proyectos PI Q002/11 (SCCyT-UNNE), PIP 11220120100150 (CONICET) y PIP 001318 (CONICET).

\section{Referencias}

Ameghino, F. (1889). Contribución al conocimiento de los mamíferos fósiles de la República Argentina. Actas Academia Nacional de Ciencias de Córdoba, 6: 32+1028 y Atlas de 98 láms.

Bergqvist, L.P. (1993). Jazimentos pleistocenicos do Estado da Paraíba e seus fósseis. Revista Nordestina de Biología, 8(2): 143-158.

Burmeister, G. (1866). Lista de los mamíferos fósiles del terreno diluviano. Anales del Museo Público de Buenos Aires, 1: 121-232.

Castellanos, A. (1937). Los sedimentos del Pampeano inferior y del Araucano en el Valle de Los Reartes (Sierra de Córdoba). Publicaciones de la Facultad de Ciencias Matemáticas, Físico-Químicas y Naturales aplicadas a la Industria, 6: 1-110.

Castellanos, A. (1942). A propósito de los géneros Plohophorus, Nopachthus y Panochthus (3a. parte). Publicaciones del Instituto de Fisiografía y Geología, 11: 413-592.

Cione, A.L.; Tonni, E.P.; Bond, M.; Carlini, A.A.; Pardiñas, U.F.J.; Scillato-Yané, G.J.; Verzi, D. \& Vucetich, M.G. (1999). Ocurrence charts of Pleistocene mammals in the Pampean area, eastern Argentina. In: Quaternary of South America and Antarctic Peninsula, Special 
Volume 12, Quaternary Vertebrate Paleontology in South America (Tonni, E.P. \& Cione A.L., eds.). Balkema, Rotterdam, 53-73.

Cione, A.L. \& Tonni, E.P. (2005). Bioestratigrafía basada en mamíferos del Cenozoico Superior de la Provincia de Buenos Aires, Argentina. Relatorio del XVI Congreso Geológico Argentino, La Plata, 184-200.

Fariña, R.A. (2000). Física y Matemáticas para reconstruir la vida en el pasado. Actas de Fisiología, 6: 45-70.

González Ruiz, L.R.; Zurita, A.E.; Fleagle, J.; ScillatoYané, G.J.; Dozo, M.T. \& Zamorano, M. (2011). The southernmost record of a Neuryurini Hoffstetter, 1958 (Mammalia, Xenarthra, Glyptodontidae). Palaeontologische Zeitschrift, 85(2): 155-161. http:// dx.doi.org/10.1007/s12542-010-0083-8.

Hoffstetter, R. (1955). Sur le genotype de Glyptodon Owen. Bulletin du Muséum National d'Historie Naturelle, 27(5): 408-413.

Lydekker, R. (1895). Contributions to a Knowledge of the Fossil Vertebrates of Argentina. 2. The Extinct Edentates of Argentina. Anales del Museo de La Plata (Paleontología Argentina III), 3: 1-118.

Mones, A. (1994). Las vicisitudes del género Panochthus Burrmeister, 1866 (Mammalia: Cingulata: Glyptodontidae). Comunicaciones Paleontológicas del Museo de Historia Natural de Montevideo, 2(27): 79-85.

Moreira, L.E. (1965). Notas previas sobre nova espécie de mamífero fóssil do Estado do Caerá, Brasil. Hy Hy Té, Revista de Facultad da Faculdade de Filosofía do Crato, 2: 41-49.

Moreira, L.E. (1971). Os gliptodontes do Nordeste do Brasil. Anais da Academia Brasileira de Ciencias, 43: 529-552.

Owen, R. (1838). Fossil Mammalia (1). In: The Zoology of the voyage of HSM Beagle (Darwin C., ed.), $40 \mathrm{pp}$.

Owen, R. (1839). Note on the Glyptodon. In: Buenos Aires and the Provinces of the Rio de La Plata (Parish, W., ed.), John Murray, Londres, 1-178.

Owen, R. (1845). Descriptive and illustrated catalogue of the fossil organic remains of Mammalia and Aves. Museum of the Royal College of Surgeons of London, London, $391 \mathrm{pp}$.

Owen, R. (1847). Notice of some fossil Mammalia of South America. 16th Meeting of the British Association for the Advancement of Science, Southampton, 65-67.

Porpino, K.O. \& Bergqvist, L.P. (2002). Novos achados de Panochthus (Mammalia, Cingulata, Glyptodontoidea) no Nordeste do Brasil. Revista Brasileira de Paleontologia, 4: 51-62.

Scillato-Yané, G.J.; Carlini, A.A.; Vizcaíno, S.F. \& OrtizJaureguizar, E. (1995). Xenarthra. In: Evolución biológica y climática de la región Pampeana durante los últimos cinco millones de años. Un ensayo de correlación con el Mediterraneo Occidental (Alberdi,
M.T.; Tonni, E.P. \& Leone, G., eds). Monografías del CSIC, España, 183-209.

Scillato-Yané, G.J. \& Carlini, A.A. 1998. Un Gigantesco Gliptodonte en los Alrededores de la Ciudad de La Plata. Revista Museo de La Plata, 11(2): 45-48.

Soibelzon, L.H.; Zamorano, M.; Scillato-Yané, G.J.; Piazza, D.; Rodríguez, S.; Soibelzon, E.; Tonni, E.P.; San Cristóbal, J. \& Beilinson, E. 2012. Un Glyptodontidae de gran tamaño en el Holoceno temprano de la región Pampeana. Revista Brasileira de Paleontologia, 15(1): 113-122. http://dx.doi.org/10.4072/ rbp.2012.1.09.

Zamorano, M. (2012a). Los Panochthini (Xenarthra, Glyptodontidae): Sistemática y evolución. Tesis Doctoral, Universidad Nacional de La Plata, 269 pp.

Zamorano (2012b) Los Panochthini (Xenarthra, Glyptodontidae): Sistemática y evolución. Mastozoología Neotropical, 19(2): 382-384.

Zamorano, M.; Scillato-Yané, G.J.; González Ruiz, L.R. \& Zurita, A.E. (2011). Revisión de los géneros Nopachtus Ameghino y Phlyctaenopyga Cabrera (Xenarthra, Glyptodontidae, Hoplophorinae) del Mioceno tardío y Plioceno de Argentina. Revista del Museo Argentino de Ciencias Naturales " Bernardino Rivadavia", 13: 59-68.

Zamorano, M.; Mones, A. \& Scillato-Yané, G.J. (2012). Redescripción y designación de un neotipo de Panochthus tuberculatus (Owen) (Mammalia: Cingulata: Glyptodontidae). Revista Brasileira de Paleontologia, 15(1): 105-112. http://dx.doi. org/10.4072/rbp.2012.1.10.

Zurita, A.E. \& Ferrero, B. (2009). Una nueva especie de Neuryurus Ameghino (Mammalia, Glyptodontidae) en el Pleistoceno tardío de la Mesopotamia de Argentina. El registro más completo del género fuera de la región Pampeana. Geobios, 42: 663-673. http:// dx.doi.org/10.1016/j.geobios.2009.03.003.

Zurita, A.E.; Soibelzon, E.; Scillato-Yané, G.J. \& Cenizo, M. (2009). The earliest record of $\mathrm{Neu}$ ryurus Ameghino (Mammalia, Glyptodontidae, Hoplophorinae). Alcheringa, 33: 32-37. http:// dx.doi.org/10.1080/03115510802618250.

Zurita, A.E.; Zamorano, M.; Scillato-Yané, G.J.; GonzálezRuiz, L.R.; Rivas Durán, B. \& Céspedez Paz, R. (2011a). An exceptional Pleistocene specimen of Panochthus Burmeister (Xenarthra, Glyptodontoidea) from Bolivia: its contribution to the understanding of the early-middle Pleistocene Panochthini. Comptes Rendus Palevol, 10: 655-664. http://dx.doi. org/10.1016/j.crpv.2011.08.002.

Zurita, A.E.; Oliva, C.; Dondas, A.; Soibelzon, E. \& Isla, F.I. (2011b). El registro más completo de un Hoplophorini (Xenarthra: Glyptodontidae) para los Pisos/Edades Chapadmalalense-Marplatense (Plioceno tardío-Pleistoceno temprano). Revista del Museo Argentino de Ciencias Naturales "Bernardino Rivadavia", 13(1): 69-75. 